Document downloaded from:

http://hdl.handle.net/10251/120546

This paper must be cited as:

Cordero Barbero, A.; Guasp, L.; Torregrosa Sánchez, JR. (2018). CMMSE2017: On two classes of fourth- and seventh-order vectorial methods with stable behavior. Journal of Mathematical Chemistry. 56(7):1902-1923. https://doi.org/10.1007/s10910-017-0814-0

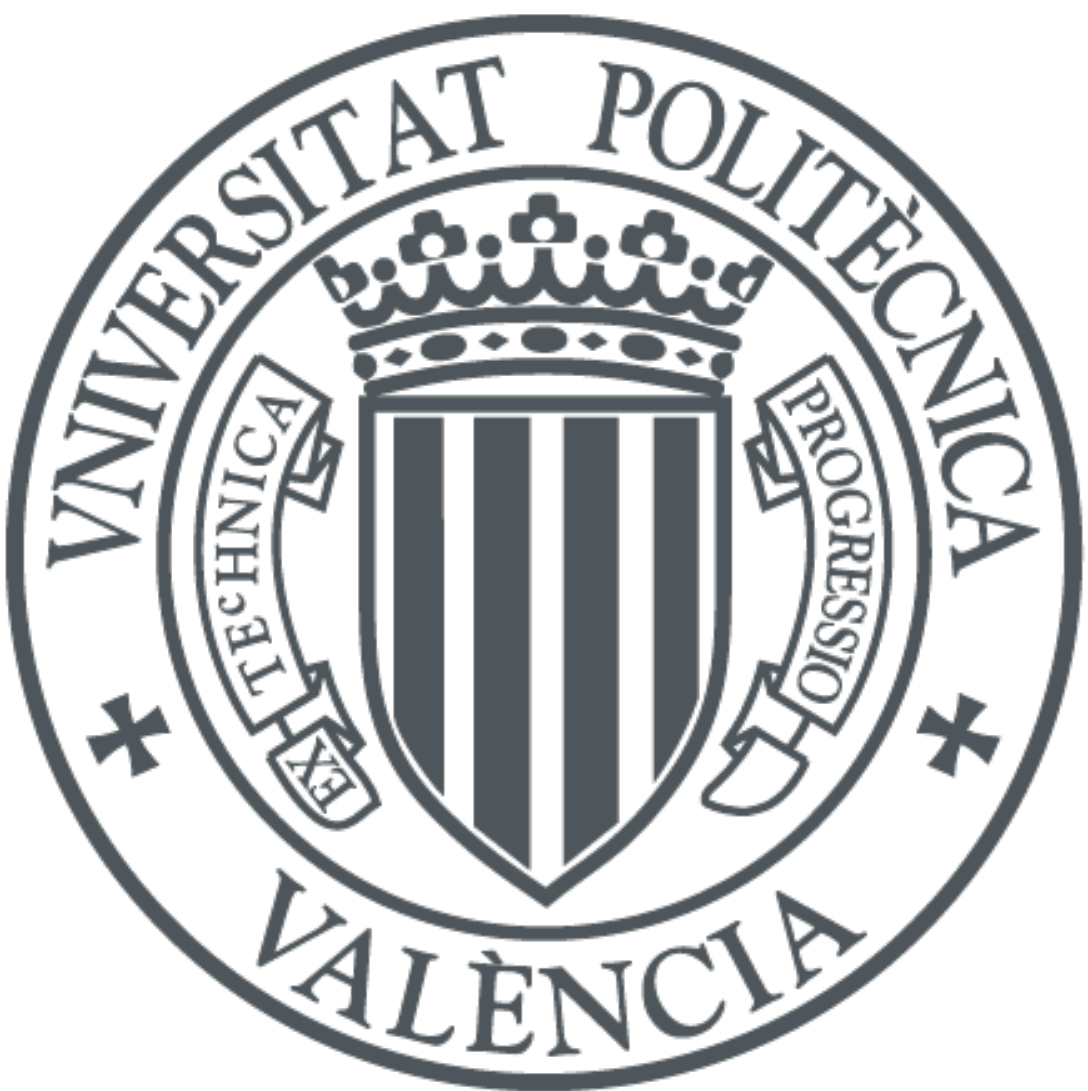

The final publication is available at

http://doi.org/10.1007/s10910-017-0814-0

Copyright Springer-Verlag

Additional Information 
Dear Author,

Here are the proofs of your article.

- You can submit your corrections online, via e-mail or by fax.

- For online submission please insert your corrections in the online correction form. Always indicate the line number to which the correction refers.

- You can also insert your corrections in the proof PDF and email the annotated PDF.

- For fax submission, please ensure that your corrections are clearly legible. Use a fine black pen and write the correction in the margin, not too close to the edge of the page.

- Remember to note the journal title, article number, and your name when sending your response via e-mail or fax.

- Check the metadata sheet to make sure that the header information, especially author names and the corresponding affiliations are correctly shown.

- Check the questions that may have arisen during copy editing and insert your answers/ corrections.

- Check that the text is complete and that all figures, tables and their legends are included. Also check the accuracy of special characters, equations, and electronic supplementary material if applicable. If necessary refer to the Edited manuscript.

- The publication of inaccurate data such as dosages and units can have serious consequences. Please take particular care that all such details are correct.

- Please do not make changes that involve only matters of style. We have generally introduced forms that follow the journal's style.

Substantial changes in content, e.g., new results, corrected values, title and authorship are not allowed without the approval of the responsible editor. In such a case, please contact the Editorial Office and return his/her consent together with the proof.

- If we do not receive your corrections within $\mathbf{4 8}$ hours, we will send you a reminder.

- Your article will be published Online First approximately one week after receipt of your corrected proofs. This is the official first publication citable with the DOI. Further changes are, therefore, not possible.

- The printed version will follow in a forthcoming issue.

\section{Please note}

After online publication, subscribers (personal/institutional) to this journal will have access to the complete article via the DOI using the URL: http://dx.doi.org/[DOI].

If you would like to know when your article has been published online, take advantage of our free alert service. For registration and further information go to: http://www.link.springer.com.

Due to the electronic nature of the procedure, the manuscript and the original figures will only be returned to you on special request. When you return your corrections, please inform us if you would like to have these documents returned. 


\section{Metadata of the article that will be visualized in OnlineFirst}

\begin{tabular}{|c|c|c|}
\hline \multicolumn{3}{|c|}{ Please note: Images will appear in color online but will be printed in black and white } \\
\hline ArticleTitle & \multicolumn{2}{|c|}{ CMMSE2017: On two classes of fourth- and seventh-order vectorial methods with stable behavior } \\
\hline \multicolumn{3}{|l|}{ Article Sub-Title } \\
\hline Article CopyRight & \multicolumn{2}{|c|}{$\begin{array}{l}\text { Springer International Publishing AG } \\
\text { (This will be the copyright line in the final PDF) }\end{array}$} \\
\hline Journal Name & \multicolumn{2}{|c|}{ Journal of Mathematical Chemistry } \\
\hline \multirow[t]{12}{*}{ Corresponding Author } & Family Name & Cordero \\
\hline & Particle & \\
\hline & Given Name & Alicia \\
\hline & Suffix & \\
\hline & Division & Instituto de Matemática Multidisciplinar \\
\hline & Organization & Universitat Politècnica de València \\
\hline & Address & Camino de Vera, s/n, 46022, Valencia, Spain \\
\hline & Phone & \\
\hline & Fax & \\
\hline & Email & acordero@mat.upv.es \\
\hline & URL & \\
\hline & ORCID & http://orcid.org/0000-0002-7462-9173 \\
\hline \multirow[t]{12}{*}{ Author } & Family Name & Guasp \\
\hline & Particle & \\
\hline & Given Name & Lucía \\
\hline & Suffix & \\
\hline & Division & Instituto de Matemática Multidisciplinar \\
\hline & Organization & Universitat Politècnica de València \\
\hline & Address & Camino de Vera, s/n, 46022, Valencia, Spain \\
\hline & Phone & \\
\hline & Fax & \\
\hline & Email & luguaal@ade.upv.es \\
\hline & URL & \\
\hline & ORCID & \\
\hline \multirow[t]{10}{*}{ Author } & Family Name & Torregrosa \\
\hline & Particle & \\
\hline & Given Name & Juan R. \\
\hline & Suffix & \\
\hline & Division & Instituto de Matemática Multidisciplinar \\
\hline & Organization & Universitat Politècnica de València \\
\hline & Address & Camino de Vera, s/n, 46022, Valencia, Spain \\
\hline & Phone & \\
\hline & Fax & \\
\hline & Email & jrtorre@mat.upv.es \\
\hline
\end{tabular}


URL

ORCID

\begin{tabular}{ll}
\hline Schedule & $\begin{array}{l}\text { Received } \\
\text { Revised } \\
\text { Accepted }\end{array}$ \\
\hline Abstract & $\begin{array}{l}\text { A family of fourth-order iterative methods without memory, for solving nonlinear systems, and its seventh- } \\
\text { order extension, are analyzed. By using complex dynamics tools, their stability and reliability are studied } \\
\text { by means of the properties of the rational function obtained when they are applied on quadratic } \\
\text { polynomials. The stability of their fixed points, in terms of the value of the parameter, its critical points } \\
\text { and their associated parameter planes, etc. give us important information about which members of the } \\
\text { family have good properties of stability and whether in any of them appear chaos in the iterative process. } \\
\text { The conclusions obtained in this dynamical analysis are used in the numerical section, where an } \\
\text { academical problem and also the chemical problem of predicting the diffusion and reaction in a porous } \\
\text { catalyst pellet are solved. }\end{array}$ \\
\hline Keywords (separated by '-') & $\begin{array}{l}\text { Nonlinear system of equations - Iterative method - Dynamical and Parameter planes - Stability } \\
\text { Footnote Information }\end{array}$ \\
$\begin{array}{l}\text { This research was partially supported by Ministerio de Economía y Competitividad MTM2014-52016- } \\
\text { C02-2-P and Generalitat Valenciana PROMETEO/2016/089. } \\
\text { This is one of several papers published together in Journal of Mathematical Chemistry on the "Special } \\
\text { Issue: CMMSE 2017". }\end{array}$ \\
\hline
\end{tabular}




\title{
CMMSE2017: On two classes of fourth- and seventh-order vectorial methods with stable behavior
}

\author{
Alicia Cordero ${ }^{1}$ (D) - Lucía Guasp ${ }^{1}$. \\ Juan R. Torregrosa ${ }^{1}$
}

Received: 25 July 2017 / Accepted: 21 September 2017

(C) Springer International Publishing AG 2017

\begin{abstract}
A family of fourth-order iterative methods without memory, for solving nonlinear systems, and its seventh-order extension, are analyzed. By using complex dynamics tools, their stability and reliability are studied by means of the properties of the rational function obtained when they are applied on quadratic polynomials. The stability of their fixed points, in terms of the value of the parameter, its critical points and their associated parameter planes, etc. give us important information about which members of the family have good properties of stability and whether in any of them appear chaos in the iterative process. The conclusions obtained in this dynamical analysis are used in the numerical section, where an academical problem and also the chemical problem of predicting the diffusion and reaction in a porous catalyst pellet are solved.
\end{abstract}

Keywords Nonlinear system of equations - Iterative method · Dynamical and Parameter planes · Stability

This is one of several papers published together in Journal of Mathematical Chemistry on the "Special Issue: CMMSE 2017”.

This research was partially supported by Ministerio de Economía y Competitividad MTM2014-52016-C02-2-P and Generalitat Valenciana PROMETEO/2016/089.

$\bowtie$ Alicia Cordero

acordero@mat.upv.es

Lucía Guasp

luguaal@ade.upv.es

Juan R. Torregrosa

jrtorre@mat.upv.es

1 Instituto de Matemática Multidisciplinar, Universitat Politècnica de València,

Camino de Vera, s/n, 46022 Valencia, Spain 


\section{Introduction}

To find the solution $x^{*}$ of a nonlinear equation $f(x)=0, f: I \subseteq \mathbb{R} \rightarrow \mathbb{R}$, or a nonlinear system $F(x)=0, F: D \subseteq \mathbb{R}^{n} \rightarrow \mathbb{R}^{n}$, is a classical and difficult problem with many applications in Science, specifically in Chemistry, and Engineering. The design and analysis of the stability properties of fixed point iterative schemes for solving equations and systems of nonlinear equations is an important and challenging task in the field of Numerical Analysis. Many problems from Chemistry consist in finding chemical potentials that are basic for studying other thermodynamic properties: the modeling of such potential leads to nonlinear integral equations that can be reduced to a set of nonlinear algebraic equations (see [16] for example). In the reaction-diffusion equations that arise in autocatalytic chemical reactions (see [15]), iterative methods can be applied; also in the analysis of electronic structure of the hydrogen atom inside strong magnetic fields (see [12]). Moreover, numerical performance of some chemical problems allows us to check the models of observable phenomena [13].

Recently, many researchers have dedicated their effort to design iterative methods for solving these type of problems, see for example [1] and [18] and the references therein. However, when a whole family of iterative procedures have similar numerical characteristics, as the order of convergence, optimality, ..., one important aspect to be taken into account is the stability of the involved schemes, that is, their dependence on the initial estimations and their tendency to be "attracted" by false solutions. These aims can be managed by analyzing the dynamical behavior of the rational operator associated to the iterative method on low degree polynomials, as have been done by other authors in, for example [2-5, 8, 14, 17].

Our goal in this paper is to carry out a dynamical study of a parametric family of iterative methods designed for solving nonlinear systems of equations $F(x)=0$, where $F: D \subseteq \mathbb{R}^{n} \rightarrow \mathbb{R}^{n}, n \geq 1$. The idea for constructing this class appears in [9] where by using the method of undetermined parameters, a method of order 5 with three steps is presented. Also a general extension of order $p+3$, where $p \geq 5$ is demonstrated when successive steps are added with the same structure. In this manuscript, we present a parametric family including that fifth-order scheme and a class of order of convergence seven including the family of order eighth from [9]. By using tools of complex dynamics, we analyze the stability of the fixed points of the rational operator that appears when our families are applied on an arbitrary second degree polynomial. The parameter plane associated to each critical point gives us important information about the stability of the elements of the family and which of them have unstable behavior.

Many problems in chemical engineering are described by means of ordinary differential equations or partial differential equations with initial or boundary conditions $[19,20]$. In the numerical section, we transform, by means of divided differences, a nonlinear boundary value problem with non-Dirichlet conditions in a nonlinear system, whose solution is an approximation of the solution of the boundary value problem in a set of discrete points of the domain. This problem allows us to predict the diffusion and reaction in a porous catalyst pellet.

In this manuscript we separate the analysis of the stability of two high-order families of iterative methods in two distinct sections: in Sect. 2, the behavior of the rational 
function related to the fourth-order family is made, by calculating their fixed and critical points, studying the stability of these fixed points, calculating the parameter plane associated to the family and representing some dynamical planes describing different behavior: stability, periodic orbits, .... In Sect. 3, a seventh-order parametric family with one element of order eight is proposed and their stability properties are analyzed in a analogous way. All this information will allows us to select those members of both classes with better stability properties, in order to be checked with a chemical problem on a porous catalyst pellet. Finally, some conclusions are stated.

\section{Fourth-order class: convergence and stability}

By adding a new step to Newton's method, we construct the following two-step scheme

$$
\begin{aligned}
y^{(k)}= & x^{(k)}-\left[F^{\prime}\left(x^{(k)}\right)\right]^{-1} F\left(x^{(k)}\right), \quad k=0,1, \ldots, \\
x^{(k+1)}= & y^{(k)}-\left(\alpha_{1} I+\alpha_{2}\left[F^{\prime}\left(y^{(k)}\right)\right]^{-1} F^{\prime}\left(x^{(k)}\right)\right. \\
& \left.+\alpha_{3}\left(\left[F^{\prime}\left(y^{(k)}\right)\right]^{-1} F^{\prime}\left(x^{(k)}\right)\right)^{2}\right)\left[F^{\prime}\left(y^{(k)}\right)\right]^{-1} F\left(y^{(k)}\right),
\end{aligned}
$$

where $\alpha_{1}, \alpha_{2}$ and $\alpha_{3}$ are free parameters.

The following result establishes the convergence of family (1), whose proof is similar to that presented in [9].

Theorem 1 Let $F: D \subseteq \mathbb{R}^{n} \rightarrow \mathbb{R}^{n}, n \geq 1$ be a sufficiently differentiable function in a convex set $D$ and $x^{*} \in D$ a root of $F(x)=0$. Choosing an initial approximation $x^{(0)}$ close enough to $x^{*}$, the iterative scheme defined by (1) has fourth-order convergence when $\alpha_{2}=2-2 \alpha_{1}$ and $\alpha_{3}=\alpha_{1}-1$, being $\alpha_{1}$ a free parameter. In particular, if $\alpha_{1}=\frac{5}{4}$, then method (1) has order five.

Proof In Theorem 1 of [9] it is proved that the error equation of this class is, under the hypothesis of the system and by using Taylor expansion of the involved functional evaluations around $x^{*}$,

$$
\begin{aligned}
e^{(k+1)}= & e_{y}{ }^{(k)}-\left[\alpha_{1} I+\alpha_{2}\left[F^{\prime}\left(y^{(k)}\right)\right]^{-1} F^{\prime}\left(x^{(k)}\right)\right. \\
& \left.+\alpha_{3}\left(\left[F^{\prime}\left(y^{(k)}\right)\right]^{-1} F^{\prime}\left(x^{(k)}\right)\right)^{2}\right]\left[F^{\prime}\left(y^{(k)}\right)\right]^{-1} F\left(y^{(k)}\right) \\
= & +\left(1-\alpha_{1}-\alpha_{2}-\alpha_{3}\right) C_{2} e^{(k)^{2}}+\left[2\left(-1+\alpha_{1}-\alpha_{3}\right) C_{2}^{2}\right. \\
& \left.+2\left(1-\alpha_{1}-\alpha_{2}-\alpha_{3}\right) C_{3}\right] e^{(k)^{3}}+\left[4\left(-1+\alpha_{1}-\alpha_{3}\right) C_{2} C_{3}\right. \\
& +3\left(-1+\alpha_{1}-\alpha_{3}\right) C_{3} C_{2}+\left(4-3 \alpha_{1}+3 \alpha_{2}+5 \alpha_{3}\right) C_{2}^{3} \\
& \left.+3\left(1-\alpha_{1}-\alpha_{2}-\alpha_{3}\right) C_{4}\right] e^{(k)^{4}}+O\left(e^{(k)}\right)
\end{aligned}
$$

where $C_{q}=(1 / q !)\left[F^{\prime}\left(x^{*}\right)\right]^{-1} F^{(q)}\left(x^{*}\right), q \geq 2, e^{(k+1)}=x^{(k+1)}-x^{*}$ and $e_{y}{ }^{(k)}=$ $y^{(k)}-x^{*}$. We observe that $C_{q} h^{q} \in \mathbb{R}^{n}$ since $F^{(q)}\left(x^{*}\right) \in \mathcal{L}\left(\mathbb{R}^{n} \times \cdots \times \mathbb{R}^{n}, \mathbb{R}^{n}\right)$ and 
$\left[F^{\prime}\left(x^{*}\right)\right]^{-1} \in \mathcal{L}\left(\mathbb{R}^{n}\right)$. In order to achieve order of convergence four, the coefficients of $e^{(k)^{2}}$ and $e^{(k)^{3}}$ must be simultaneously null and then parameters $\alpha_{1}, \alpha_{2}$ and $\alpha_{3}$ must satisfy

$$
\left\{\begin{array}{l}
1-\alpha_{1}-\alpha_{2}-\alpha_{3}=0 \\
-1+\alpha_{1}-\alpha_{3}=0
\end{array}\right.
$$

From this system, it is straightforward that $\alpha_{2}=2-2 \alpha_{1}$ and $\alpha_{3}=\alpha_{1}-1$ and the thesis is proved.

Once we know that all the methods of these class have, at least, order of convergence four, we want to analyze which is the relation between the values of the free parameter $\alpha_{1}$ and the stability of the corresponding iterative method? By using the tools of complex discrete dynamics, we are going to study the general convergence of the families on quadratic polynomials. To get this aim, we firstly recall some dynamical concepts (a wider revision of these aspects can be found in $[6,11]$ ). Given a rational function $R: \hat{\mathbb{C}} \rightarrow \hat{\mathbb{C}}$, where $\hat{\mathbb{C}}$ is the Riemann sphere, the orbit of a point $z_{0} \in \hat{\mathbb{C}}$ is defined as:

$$
\left\{z_{0}, R\left(z_{0}\right), R^{2}\left(z_{0}\right), \ldots, R^{n}\left(z_{0}\right), \ldots\right\} .
$$

We analyze the phase plane of the map $R$ by classifying the starting points from the asymptotic behavior of their orbits. A $z_{0} \in \hat{\mathbb{C}}$ is called a fixed point if $R\left(z_{0}\right)=z_{0}$. It is a particular case of a periodic point $z_{0}$ of period $p>1$ is a point such that $R^{p}\left(z_{0}\right)=z_{0}$ and $R^{k}\left(z_{0}\right) \neq z_{0}$, for $k<p$. However, a pre-periodic point is a point $z_{0}$ that is not periodic but there exists a $k>0$ such that $R^{k}\left(z_{0}\right)$ is periodic. Also a critical point $z_{0}$ is a point where the derivative of the rational function vanishes, $R^{\prime}\left(z_{0}\right)=0$. Moreover, a fixed point $z_{0}$ is called attractor if $\left|R^{\prime}\left(z_{0}\right)\right|<1$, superattractor if $\left|R^{\prime}\left(z_{0}\right)\right|=0$, repulsor if $\left|R^{\prime}\left(z_{0}\right)\right|>1$ and parabolic if $\left|R^{\prime}\left(z_{0}\right)\right|=1$.

The basin of attraction of an attractor $z^{*}$ is defined as:

$$
\mathcal{A}(\alpha)=\left\{z_{0} \in \hat{\mathbb{C}}: R^{n}\left(z_{0}\right) \rightarrow z^{*}, n \rightarrow \infty\right\} .
$$

The immediate basin of attraction of an attractor is the connected component of its basin of attraction that holds the attractor.

The Fatou set of the rational function $R, \mathcal{F}(R)$, is the set of points $z \in \hat{\mathbb{C}}$ whose orbits tend to an attractor (fixed point, periodic orbit or infinity). Its complement in $\hat{\mathbb{C}}$ is the Julia set, $\mathcal{J}(R)$. That means that the basin of attraction of any fixed point belongs to the Fatou set and the boundaries of these basins of attraction belong to the Julia set.

The following theorem establishes a classical result of Fatou and Julia that we use in the study of parameter space associated to the family.

Theorem 2 Let $R$ be a rational function. The immediate basin of attraction of an attracting fixed or periodic point holds, at least, a critical point.

By using this result, one can be sure to find all the stable behavior associated to a rational function $R$, by analyzing the performance of $R$ on the set of critical points. 
It is known that, if the iterative method satisfies the Scaling Theorem (and family (1) does, as it used first-order derivatives [2]), the roots of a polynomial can be transformed by an affine map with no qualitative changes on the dynamics of the family. So, we can use a generic quadratic polynomial $p(z)=(z-a)(z-b)$. The rational operator obtained when family (1) is applied on $p(z)$ has the expression:

$$
\begin{aligned}
T_{p, \alpha_{1}, a, b}(z)= & \frac{(a-z)(b-z)}{a+b-2 z}+z+(a-z)^{2}(b-z)^{2} \\
& \times\left[\frac{\left(a^{4}+b^{4}-4 a^{3} z-4 b^{3} z+4\left(1+\alpha_{1}\right) b^{2} z^{2}-8 \alpha_{1} b z^{3}\right.}{(a+b-2 z)\left(a^{2}+b^{2}-2 a z-2 b z+2 z^{2}\right)^{3}}\right. \\
& +\frac{4 \alpha_{1} z^{4}-4 a z\left(\left(-1+2 \alpha_{1}\right) b^{2}+\left(2-4 \alpha_{1}\right) b z+2 \alpha_{1} z^{2}\right)}{(a+b-2 z)\left(a^{2}+b^{2}-2 a z-2 b z+2 z^{2}\right)^{3}} \\
& \left.+\frac{\left.a^{2}\left(\left(-2+4 \alpha_{1}\right) b^{2}+\left(4-8 \alpha_{1}\right) b z+4\left(1+\alpha_{1}\right) z^{2}\right)\right)}{(a+b-2 z)\left(a^{2}+b^{2}-2 a z-2 b z+2 z^{2}\right)^{3}}\right],
\end{aligned}
$$

depending on parameter $\alpha_{1}$ and also on the roots of the polynomial $a$ and $b$.

Blanchard in [6] considered the conjugacy map $h(z)=\frac{z-a}{z-b}$, (a Möbius transformation) with the following properties:
i) $h(\infty)=1$,
ii) $h(a)=0$,
iii) $h(b)=\infty$,

and proved that, for quadratic polynomials, Newton's operator is conjugate to the rational map $z^{2}$, that is it satisfies Cayley's test (see [5]). In an analogous way, operator $T_{p, \alpha_{1}, a, b}(z)$ on quadratic polynomials is conjugated to operator $O_{\alpha_{1}}(z)$,

$$
O_{\alpha_{1}}(z)=\left(h \circ T_{p, \alpha_{1}, a, b} \circ h^{-1}\right)(z)=-z^{4} \frac{5-4 \alpha_{1}+2 z^{2}+z^{4}}{-1-2 z^{2}+-5 z^{4}+4 \alpha_{1} z^{4}} .
$$

We observe that parameters $a$ and $b$ have been obviated in $O_{\alpha_{1}}(z)$.

\subsection{Analysis of the fixed and critical points}

Firstly, we study the fixed points of the rational function $O_{\alpha_{1}}(z)$ that are not related with the original roots of the polynomial $p(z)$ (called strange fixed points), and the free critical points, that is, the critical points of $O_{\alpha_{1}}(z)$ different from 0 and $\infty$, which are associated to the roots of $p(z)$.

Fixed points of $O_{\alpha_{1}}(z)$ are the roots of equation $O_{\alpha_{1}}(z)=z$, that is, $z=0, z=\infty$ and the strange fixed points $\operatorname{ex}_{1}\left(\alpha_{1}\right)=1$ and the roots of the polynomial

$$
r\left(\alpha_{1}, z\right)=1+z+3 z^{2}+\left(-2+4 \alpha_{1}\right) z^{3}+3 z^{4}+z^{5}+z^{6},
$$

that are denoted by $e x_{i}\left(\alpha_{1}\right), i=2,3, \ldots, 7$. By analyzing the common roots of the polynomials involved in numerator and denominator of rational function $O_{\alpha_{1}}(z)$, it can be also stated which are the values of parameter $\alpha_{1}$ such that the number of fixed 
points decreases, as these are possible elements of the family with better stability, to be analyzed later deeply. These results are summarized in the following result.

Proposition 1 Rational function $O_{\alpha_{1}}(z)$ has seven strange fixed points, ex $x_{1}\left(\alpha_{1}\right)=$ 1 (if $\alpha_{1} \neq 2$ ) and the roots of the polynomial $r\left(\alpha_{1}, z\right)$, denoted by ex $x_{i}\left(\alpha_{1}\right), i=$ $2,3, \ldots, 7$, except in the following cases:

(i) If $\alpha_{1}=1$, then the operator is $O_{1}(z)=z^{4}$, so there are no strange fixed points, and the corresponding element of family (1) satisfies Cayley test.

(ii) If $\alpha_{1}=2$, then the operator is $O_{2}(z)=-z^{4} \frac{3+z^{2}}{1+3 z^{2}}$. There are only five strange fixed points as fixed point equation is reduced to $z(z+1)\left(1-z+4 z^{2}-z^{3}+z^{4}\right)=0$. (iii) If $\alpha_{1}=-2, O_{-2}(z)=z^{4} \frac{13+2 z^{2}+z^{4}}{1+2 z^{2}+13 z^{4}}$ and there are only five strange fixed point (as ex $x_{2}\left(\alpha_{1}\right)=\operatorname{ex}_{3}\left(\alpha_{1}\right)=1$ ), that correspond to the roots of polynomial $1+3 z+$ $8 z^{2}+3 z^{3}+z^{4}$.

Of course, as the order of the iterative method is greater than two, $z=0$ and $z=\infty$ are superattracting fixed points but, which is the character of the rest of fixed points? To answer this question, $O_{\alpha_{1}}^{\prime}\left(e x_{i}\left(\alpha_{1}\right)\right), i=1,2, \ldots, 7$ must be analyzed. In case of $e x_{1}\left(\alpha_{1}\right)$, it is easy to check that $O_{\alpha_{1}}^{\prime}(1)=-\frac{4}{\alpha_{1}-2}$, so the following result can be stated.

Theorem 3 The character of the strange fixed point ex $x_{1}\left(\alpha_{1}\right)=1$ of the rational function $O_{\alpha_{1}}(z), \alpha_{1} \neq 2$, is as follows:

(i) If $\left|\alpha_{1}-2\right|>4$, then ex $x_{1}\left(\alpha_{1}\right)=1$ is an attractor.

(ii) When $\left|\alpha_{1}-2\right|=4$, ex $x_{1}\left(\alpha_{1}\right)=1$ is a parabolic point.

(iii) If $\left|\alpha_{1}-2\right|<4$, then ex $x_{1}\left(\alpha_{1}\right)=1$ is a repulsor.

In Fig. 1, the stability function $\left|O_{\alpha_{1}}^{\prime}(1)\right|$ is represented in the complex plane, showing a circle where this strange fixed point is repulsive, that is, where the original methods will not diverge. Moreover, it can be checked that strange fixed points $e x_{i}\left(\alpha_{1}\right), i=$ $2,3,4,5$ are repulsive for all complex values of $\alpha_{1}$ and $e x_{6}\left(\alpha_{1}\right)$ and $e x_{7}\left(\alpha_{1}\right)$ are simultaneously attracting in a region close to the origin. The analysis of the stability of strange fixed points $e x_{i}\left(\alpha_{1}\right), i=2,3,4,5$ shows that they are repulsive for any value of the parameter. As they have not explicit expressions, we plot in Fig. 2, their stability regions of all strange fixed points $e x_{i}\left(\alpha_{1}\right), i=1,2, \ldots, 7$ (Fig. 3).

As we have stated previously, a classical result from Julia and Fatou establishes that there is, at least, one critical point associated with each invariant Fatou component. Due to the order of convergence of the methods under study, it is clear that $z=0$ and $z=\infty$ (related to the roots of the polynomial by means of Möbius map) are critical points and give rise to their respective Fatou components, but there exist in the family some free critical points, some of them depending on the value of the parameter, that can be held in other components of Fatou set and give rise to other attracting behavior.

By analyzing the equation $O_{\alpha_{1}}^{\prime}(z)=0$, we obtain that it can be reduced to

$$
z^{3}\left(1+z^{2}\right)^{2}\left(-5+2 z^{2}-5 z^{4}+4 \alpha_{1}\left(1-z^{2}+z^{4}\right)\right)=0 .
$$

Then, $z=i$ and $z=-i$ are always free critical points but they must not be taken into account to analyze the quantity of possible stable behaviors, in terms of Fatou-Julia 


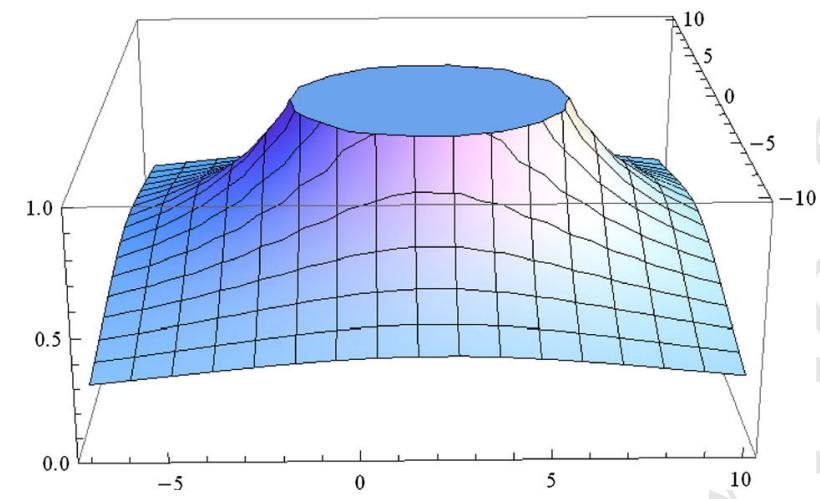

Fig. $1\left|O_{\alpha_{1}}^{\prime}(1)\right|$

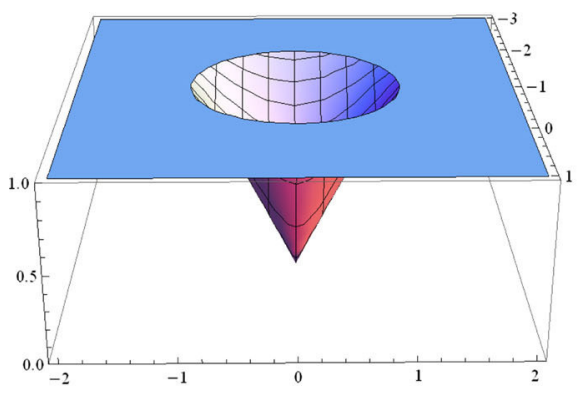

(a)

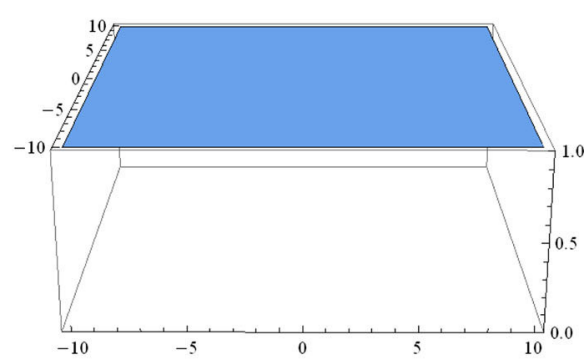

(b)

Fig. 2 Stability regions of some strange fixed points. a $\left|O_{\alpha_{1}}^{\prime}\left(\operatorname{ex}_{i}\left(\alpha_{1}\right)\right)\right|, i=6,7, \mathbf{b}\left|O_{\alpha_{1}}^{\prime}\left(e x_{i}\left(\alpha_{1}\right)\right)\right|, i=$ $2,3,4,5$

Theorem, as both are pre-images of $z=1$, that is a strange fixed point. As both critical points "converge" to $z=1$, they will be the responsible of its attractive behavior, when it happens (see Theorem 3).

On the other hand, other four free critical points appear as roots of polynomial $-5+4 \alpha_{1}+\left(2-4 \alpha_{1}\right) z^{2}+\left(-5+4 \alpha_{1}\right) z^{4}$ that can be immediately obtained by the change of variables $t=z^{2}$, as

$$
\begin{aligned}
& t_{1}=\frac{1+2 \sqrt{3} \sqrt{-\left(-2+\alpha_{1}\right)\left(-1+\alpha_{1}\right)}-2 \alpha_{1}}{5-4 \alpha_{1}} \text { and } \\
& t_{2}=\frac{-1+2 \sqrt{3} \sqrt{-\left(-2+\alpha_{1}\right)\left(-1+\alpha_{1}\right)}+2 \alpha_{1}}{-5+4 \alpha_{1}},
\end{aligned}
$$

resulting the rest of free critical points $z= \pm \sqrt{t_{1}}$ and $z= \pm \sqrt{t_{2}}$. These results have been summarized in the following proposition.

Proposition 2 The number of free critical points of rational function $O_{\alpha_{1}}(z)$ corresponding to family (1) is: 


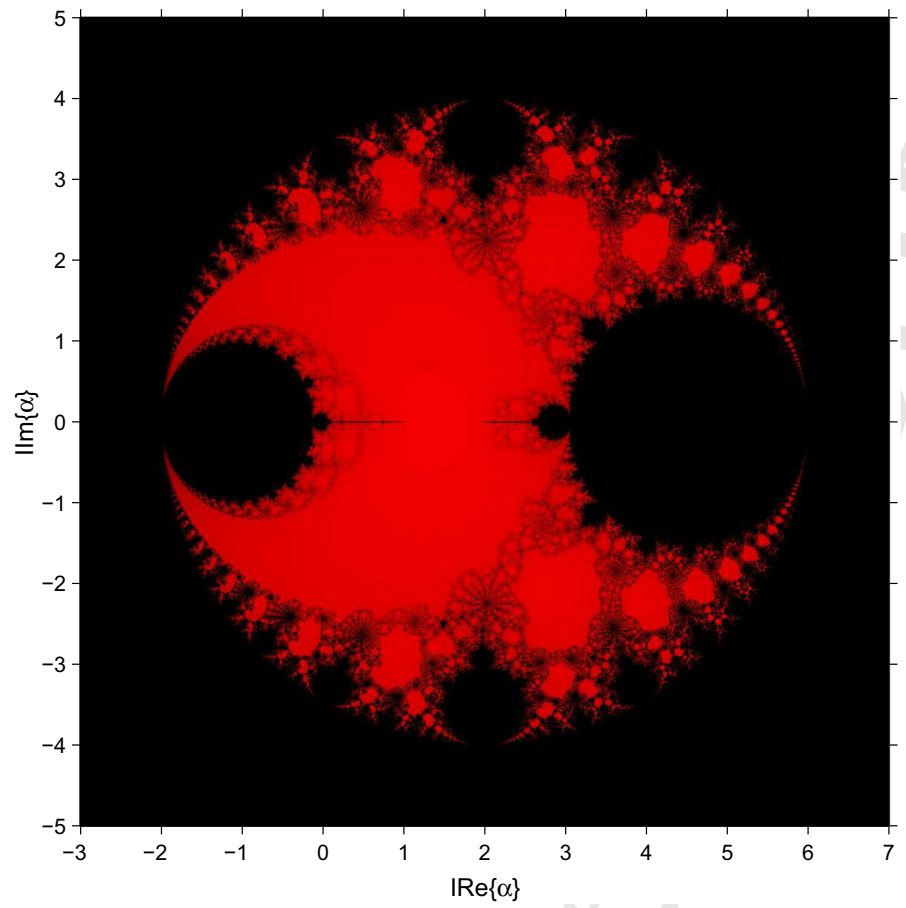

Fig. 3 Parameter plane $P_{1}$ associated to $c r_{i}\left(\alpha_{1}\right), i=3,4,5,6$

(a) None, if $\alpha_{1}=1$, as there is no free critical points of operator $O_{\alpha_{1}}(z)$.

(b) Two, if $\alpha_{1}=2$ or $\alpha_{1}=\frac{5}{4}$, as in this case $z=-i$ and $z=i$ are the only free critical points. Moreover, the order of convergence of the method corresponding to $\alpha_{1}=\frac{5}{4}$ increases to six as $O_{\frac{5}{4}}(z)=z^{6} \frac{\left(2+z^{2}\right)}{1+2 z^{2}}$.

(c) In any other case, the free critical points are:

$$
\begin{aligned}
& \operatorname{cr}_{1}\left(\alpha_{1}\right)=-i \\
& \operatorname{cr}_{2}\left(\alpha_{1}\right)=i, \\
& \operatorname{cr}_{3}\left(\alpha_{1}\right)=-\sqrt{\frac{1-2 \alpha_{1}+2 \sqrt{3} \sqrt{-2+3 \alpha_{1}-\alpha_{1}^{2}}}{5-4 \alpha_{1}}}=-\operatorname{cr}_{4}\left(\alpha_{1}\right)=\frac{1}{c r_{5}\left(\alpha_{1}\right)}, \\
& \operatorname{cr}_{6}\left(\alpha_{1}\right)=\sqrt{\frac{-1+2 \alpha_{1}+2 \sqrt{3} \sqrt{-2+3 \alpha_{1}-\alpha_{1}^{2}}}{-5+4 \alpha_{1}}}=-\operatorname{cr}_{5}\left(\alpha_{1}\right)=\frac{1}{c r_{4}\left(\alpha_{1}\right)} .
\end{aligned}
$$

Let us remark that $c r_{1}\left(\alpha_{1}\right)$ and $c r_{2}\left(\alpha_{1}\right)$ are pre-images of $z=1$ and $c r_{3}\left(\alpha_{1}\right)$ and $\operatorname{cr}_{5}\left(\alpha_{1}\right)$ are conjugated, as well as $c r_{4}\left(\alpha_{1}\right)$ and $\operatorname{cr}_{6}\left(\alpha_{1}\right)$. Moreover, the rational function $O_{\alpha_{1}}(z)$ has only even powers and it is satisfied $\operatorname{cr}_{6}\left(\alpha_{1}\right)=-c r_{5}\left(\alpha_{1}\right)$ and $c r_{3}\left(\alpha_{1}\right)=-c r_{4}\left(\alpha_{1}\right)$. So, we only have one independent free critical point, whose 
asymptotic behavior will determine if can be some attracting elements in the phase space, apart from those coming from the roots of the polynomial.

\subsection{The parameter and dynamical planes}

The parameter space associated with an independent free critical point of operator is obtained by associating each point of the complex plane with a value of $\alpha_{1}$, i.e., with an element of family. Every value of the parameter belonging to the same connected component of the parameter space gives rise to subsets of schemes of the family with similar dynamical behavior. So, it is interesting to find regions of the parameter plane as much stable as possible, because these values of the parameter will give us the best members of the family in terms of numerical stability.

When we consider the independent free critical point of operator $O_{\alpha_{1}}(z)$ as a starting point of the iterative scheme of the family associated to each complex value of $\alpha_{1}$, we paint this point of the complex plane in red if the method converges to any of the roots (zero and infinity) and they are black in other cases. The color used is brighter when the number of iterations is lower. Then, the parameter plane $P_{1}$ is obtained. A mesh of $1000 \times 1000$ points has been used, 500 has been the maximum number of iterations involved and $10^{-3}$ the tolerance used as a stopping criterium (see [7]).

We obtain an only parameter plane due to the fact that $c r_{4}\left(\alpha_{1}\right)$ is equal in module to ${ } r_{6}\left(\alpha_{1}\right)$ and the operator's powers are even numbers. We can observe that the best real values of the parameter $\alpha_{1}$ are between 1 and 2, as the only allowed convergence of the methods is to the roots of the original polynomial (to 0 and $\infty$ after Möbius transformation), and a complex region of values of the parameter associated to stable elements of the family (in red in the parameter plane) is identified.

Now we show, by means of dynamical planes, the qualitative behavior of the different elements of the family. We select these elements by using the conclusions obtained by analyzing the parameter plane and the stability analysis made on fixed points.

The dynamical plane associated to a value of the parameter, that is, obtained by iterating an element of family, is generated by using each point of the complex plane as initial estimation (we have used a mesh of $400 \times 400$ points). We paint in blue the points whose orbit converges to infinity, in orange the points converging to zero (with a tolerance of $10^{-3}$ ), in other colors (green, red, etc.) those points whose orbit converges to one of the strange fixed points (all fixed points appear marked as a white star in the figures) and in black if it reaches the maximum number of 40 iterations without converging to any of the fixed points. In Fig. 4 (obtained by using the software in [7]), we show the dynamical planes corresponding to stable values of the parameter, specifically $\alpha_{1}=1, \alpha_{1}=2$ and $\alpha_{1}=0.5$.

On the other hand, unstable behavior is found when we choose values of $\alpha_{1}$ in the black region of parameter plane. In Fig. 5, dynamical planes corresponding to values of parameter $\alpha_{1}=3, \alpha_{1}=3.5$ and $\alpha_{1}=-1.5$ are presented. In Fig. 5a, b we can observe periodic orbits of period two, while in Fig. $5 \mathrm{c}$ four basins of attraction appear, two of them corresponding to 0 and $\infty$ (associated to the roots of $p(z)$ ) and the other ones are the basins of attraction of the strange fixed points $e x_{i}\left(\alpha_{1}\right), i=5,6$, that are attracting for this value of the parameter, as $\alpha_{1}=0.5$ has been selecting in size of the disk defined by the stability function of these fixed points (see Fig. 2a). 


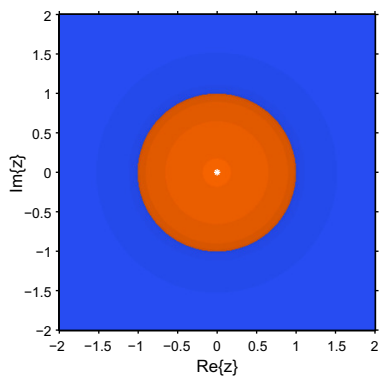

(a)

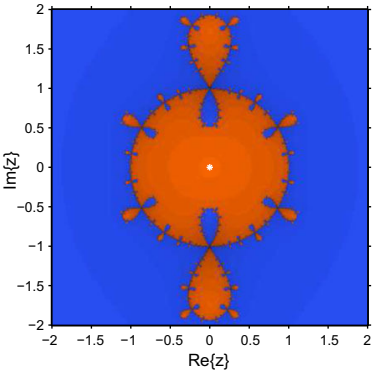

(b)

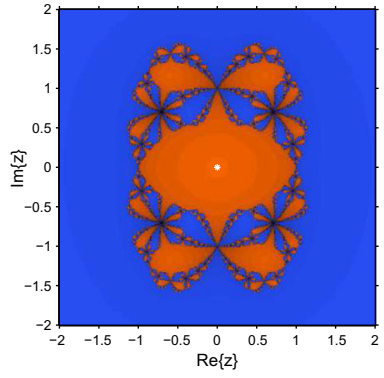

(c)

Fig. 4 Some dynamical planes with stable behavior. a $\alpha_{1}=1, \mathbf{b} \alpha_{1}=2$, $\mathbf{c} \alpha_{1}=0.5$

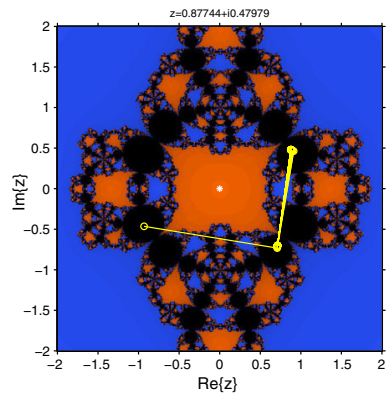

(a)

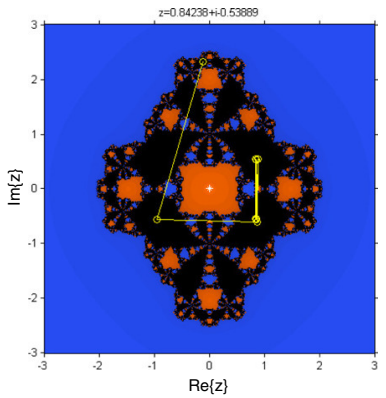

(b)

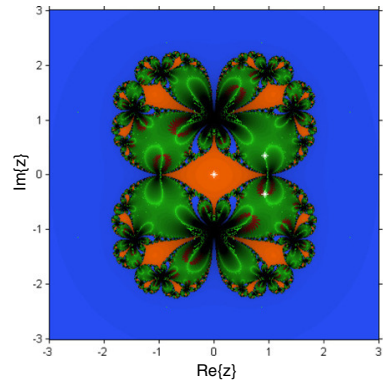

(c)

Fig. 5 Dynamical planes with unstable behavior. a $\alpha_{1}=3$, b $\alpha_{1}=3.5$, c $\alpha_{1}=-1.5$

\section{Increasing the order: How does the stability changes with the order of convergence?}

Now, our aim is to improve the order of convergence of family (1) with a new step with a similar structure as the last one of fourth-order. Once its order is stated, we analyze its stability and compare with that obtained in the previous section. We take $\alpha_{1}=\frac{5}{4}$ in (1) (fifth-order of convergence for any nonlinear function and only two critical points in the dynamical analysis that are pre-images of the strange fixed point $z=1$, on quadratic polynomials) and add one step to increase the order of the method to seven or eight, obtaining the following expression

$$
\begin{aligned}
x^{(k+1)}= & t^{(k)}-\left(\beta_{1} I+\beta_{2}\left[F^{\prime}\left(y^{(k)}\right)\right]^{-1} F^{\prime}\left(x^{(k)}\right)\right. \\
& \left.+\beta_{3}\left(\left[F^{\prime}\left(y^{(k)}\right)\right]^{-1} F^{\prime}\left(x^{(k)}\right)\right)^{2}\right)\left[F^{\prime}\left(y^{(k)}\right)\right]^{-1} F\left(t^{(k)}\right),
\end{aligned}
$$

for $k=0,1, \ldots$, where $y^{(k)}=x^{(k)}-\left[F^{\prime}\left(x^{(k)}\right)\right]^{-1} F\left(x^{(k)}\right)$ and $t^{(k)}$ are the first and second steps, respectively, of class (1). The following result gives us the values of the parameter that highly improve the order of convergence. 
Theorem 4 Let $F: D \subseteq \mathbb{R}^{n} \rightarrow \mathbb{R}^{n}, n \geq 1$ be a sufficiently differentiable function in a convex set $D$ and $x^{*} \in D$ a root of $F(x)=0$. Choosing an initial approximation $x^{(0)}$ close enough to $x^{*}$, the iterative scheme defined by (3) has seventh-order convergence when $\beta_{2}=-2\left(\beta_{1}-1\right)$ and $\beta_{3}=\beta_{1}-1$, being $\beta_{1}$ a free parameter. Specifically, if $\beta_{1}=\frac{3}{2}$ then method (3) has order eight.

In a similar way as it was made in Sect. 2, the rational function of the operator on $p(z)=(z-a)(z-b)$ is denoted by $T_{p, \beta_{1}, a, b}(z)$ and it depends on parameter $\beta_{1}$ and also on the roots of the polynomial $a$ and $b$. However, by means of the Möbius map $h(z)=\frac{z-a}{z-b}$, operator $T_{p, \beta_{1}, a, b}(z)$ is conjugated to operator $O_{\beta_{1}}(z)$ on quadratic polynomials, where

$$
\begin{aligned}
O_{\beta_{1}}(z) & =\left(h \circ T_{p, \alpha_{1}, a, b} \circ h^{-1}\right)(z) \\
& =-z^{8} \frac{\left(2+z^{2}\right)\left(6+18 z^{2}+18 z^{4}+15 z^{6}+6 z^{8}+z^{10}-4 \beta_{1}\left(1+2 z^{2}\right)\right)}{\left(1+2 z^{2}\right)\left(-1-6 z^{2}-15 z^{4}-18 z^{6}+2\left(-9+4 \beta_{1}\right) z^{8}+\left(-6+4 \beta_{1}\right) z^{10}\right)},
\end{aligned}
$$

where the parameters $a$ and $b$ have been obviated. Let us remark that, although the order of convergence of the members of the family is, in general, seven, the eighthpower of the rational function shows us that, on quadratic polynomials, the order is at least, eight. In an analogous way as it has been done for the fourth-order family in the previous section, we analyze in the following the fixed and critical points, in order to detect those elements of the class with better stability properties and compare the obtained results.

\subsection{Analysis of the fixed and critical points}

In this case, the fixed points of the operator are the roots of equation $O_{\beta_{1}}(z)=z$, that is, $z=0, z=\infty$ and the strange fixed points $e_{1}\left(\beta_{1}\right)=1$ and the roots of the polynomial $r\left(\beta_{1}, z\right)=1+z+9 z^{2}+9 z^{3}+36 z^{4}+36 z^{5}+84 z^{6}+\left(72+8 \beta_{1}\right) z^{7}+126 z^{8}+(84+$ $\left.20 \beta_{1}\right) z^{9}+126 z^{10}+\left(72+8 \beta_{1}\right) z^{11}+84 z^{12}+36 z^{13}+36 z^{14}+9 z^{15}+9 z^{16}+z^{17}+z^{18}$.

Therefore, there exist nineteen strange fixed points, except if $\beta_{1}=-\frac{208}{9}$, the rational function is there are sixteen strange fixed points.

In order to classify them depending on their asymptotic behavior, we calculate the first derivative of $O_{\beta_{1}}(z)$ :

$O_{\beta_{1}}^{\prime}(z)=-4 z^{7} \frac{\left(1+z^{2}\right)^{8}\left(2 \beta_{1}\left(8+9 z^{2}-16 z^{4}+9 z^{6}+8 z^{8}\right)-3\left(8+19 z^{2}+10 z^{4}+19 z^{6}+8 z^{8}\right)\right)}{\left(1+2 z^{2}\right)^{2}\left(-1+z^{2}\left(2 z+z^{2}\right)\left(-3-6 z^{2}-6 z^{4}+\left(-6+4 \beta_{1}\right) z^{6}\right)\right)^{2}}$,

which has only even powers, as in the fourth-order case.

As it is proven in the following result, the stability of the first strange fixed point of $O_{\beta_{1}}(z)$ depends on the value of the parameter, existing a disk in the complex plane where it is repulsive and, therefore, the original methods will not diverge (see Fig. 6). Let us remark that this area is much bigger than that obtained in case of order four.

Theorem 5 The character of the strange fixed point ex $x_{1}\left(\beta_{1}\right)=1, \beta_{1} \neq \frac{16}{3}$, is as follows: 


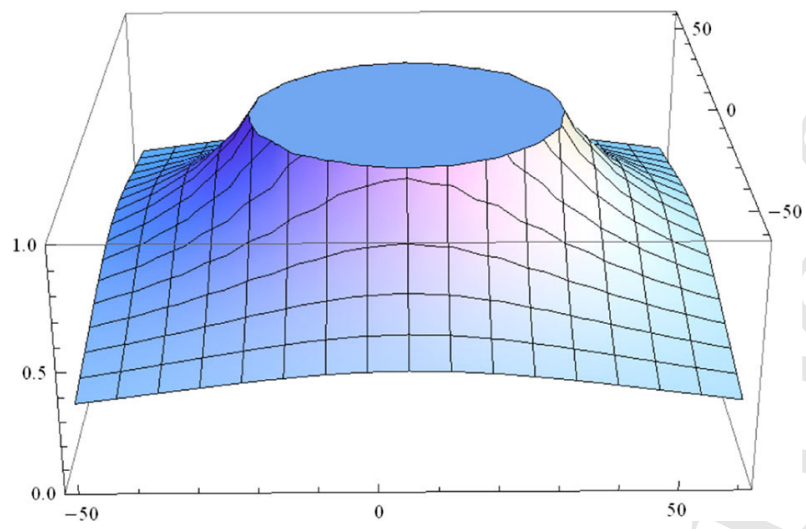

Fig. 6 Stability function $\left|O_{\beta_{1}}\left(e x_{1}\left(\beta_{1}\right)\right)\right|$

31

(i) When $\left|\beta_{1}-\frac{32}{6}\right|>\frac{256}{9}$, ex $1\left(\beta_{1}\right)=1$ is an attractor.

(ii) If $\left|\beta_{1}-\frac{32}{6}\right|=\frac{256}{9}$, ex $x_{1}\left(\beta_{1}\right)=1$ is a parabolic point.

(iii) When $\left|\beta_{1}-\frac{32}{6}\right|<\frac{256}{9}$, then ex $x_{1}\left(\beta_{1}\right)=1$ is a repulsor.

Proof It is easy to see that

$$
O_{\beta_{1}}^{\prime}(1)=\frac{256}{48-9 \beta_{1}} \text {. }
$$

So,

$$
\left|\frac{256}{48-9 \beta_{1}}\right| \leq 1 \text { is equivalent to } 256 \leq\left|48-9 \beta_{1}\right|
$$

Let us consider $\beta_{1}=a+i b$ an arbitrary complex number. Then,

$$
256^{2} \leq 48^{2}-864 a+81 a^{2}+81 b^{2} .
$$

By simplifying

$$
81 a^{2}-864 a+81 b^{2}-63232 \geq 0,
$$

that is,

$$
\left(a-\frac{32}{6}\right)^{2}+b^{2} \geq \frac{65536}{81} .
$$

Therefore,

$$
\left|O_{\beta_{1}}^{\prime}(1)\right| \leq 1 \text { if and only if }\left|\beta_{1}-\frac{32}{6}\right| \geq \frac{256}{9} .
$$




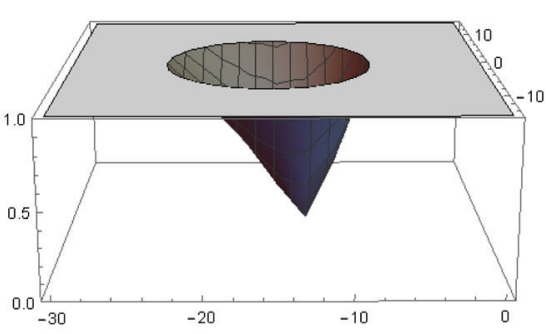

(a)

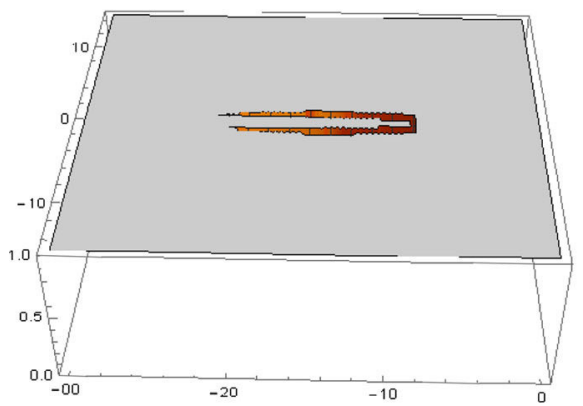

(c)

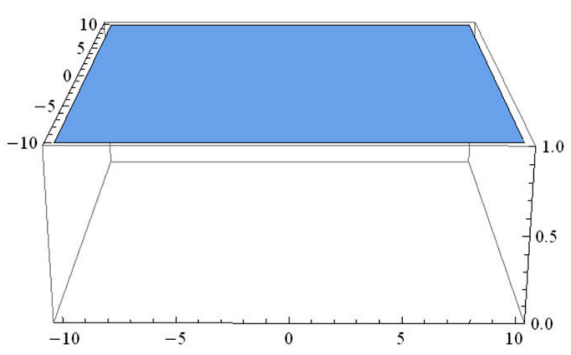

(e)

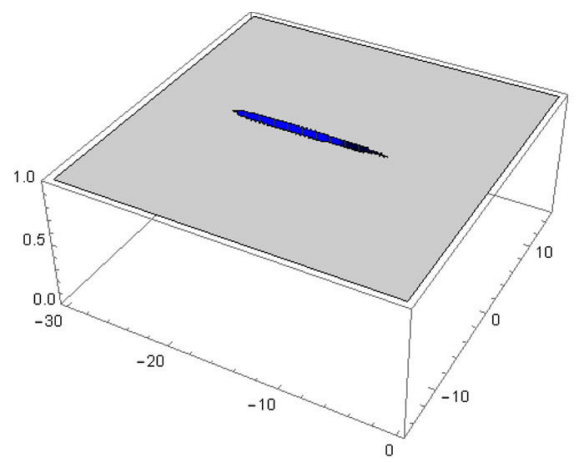

(b)

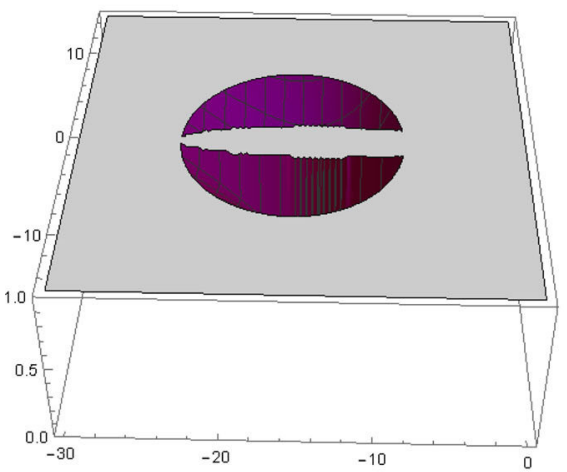

(d)

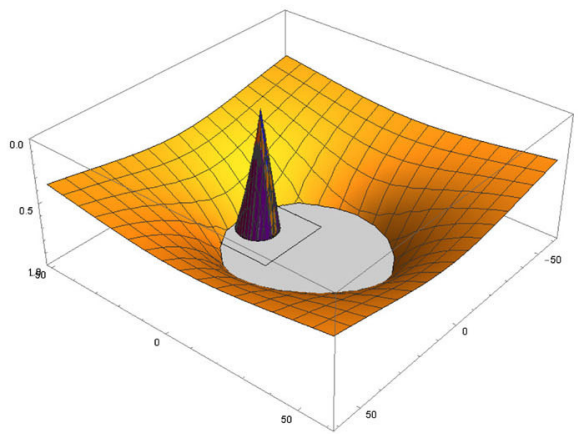

(f)

Fig. 7 Stability functions of the different strange fixed points of $O_{\beta_{1}}(z)$. a $\left|O_{\beta_{1}}\left(e x_{16}\left(\beta_{1}\right)\right)\right|$, $\mathbf{b}\left|O_{\beta_{1}}\left(\operatorname{ex}_{17}\left(\beta_{1}\right)\right)\right|, \mathbf{c}\left|O_{\beta_{1}}\left(\operatorname{ex}_{18}\left(\beta_{1}\right)\right)\right|, \mathbf{d}\left|O_{\beta_{1}}\left(\operatorname{ex}_{19}\left(\beta_{1}\right)\right)\right|, \mathbf{e}\left|O_{\beta_{1}}\left(e x_{i}\left(\beta_{1}\right)\right)\right|, \quad i \in\{2,3, \ldots, 14,15\}$, f union of the stability functions

In Fig. 7, we represent the stability regions of the rest of strange fixed points $e x_{i}\left(\beta_{1}\right)$, $i=2,3, \ldots, 19$. We observe that strange points $e x_{i}\left(\beta_{1}\right), i=2,3, \ldots, 14,15$ are repulsive for any value of the parameter $\beta_{1}$, and only four of them can be attracting in an area surrounding $\beta_{1}=-15$, with approximate radius 5 . They are shown in 
different colors (grey for $\operatorname{ex}_{16}\left(\beta_{1}\right)$, blue for $\operatorname{ex}_{17}\left(\beta_{1}\right)$, orange for $e x_{18}\left(\beta_{1}\right)$ and purple for $\operatorname{ex}_{19}\left(\beta_{1}\right)$. In this way, it is easy to observe that $\operatorname{ex}_{16}\left(\beta_{1}\right)$ is simultaneously attracting with only one of the other three points, whose union of their respective stability functions coincide with the stability function of $\operatorname{ex}_{16}\left(\beta_{1}\right)$.

On the other hand, it is clear that $z=0$ and $z=\infty$ (related to the roots of the polynomial) are critical points. The rest of critical points are found by solving the equation $O_{\beta_{1}}^{\prime}(z)=0$, that is, the roots of $\left(1+z^{2}\right)^{8}\left(2 \beta_{1}\left(8+9 z^{2}-16 z^{4}+9 z^{6}+\right.\right.$ $\left.\left.8 z^{8}\right)-3\left(8+19 z^{2}+10 z^{4}+19 z^{6}+8 z^{8}\right)\right)$. Some of them coincide with those of the fourth-order family, as the roots of $\left(1+z^{2}\right)$, that are again pre-images of $z=1$ and then do not have independent stability to be considered in the parameter planes. These and the rest of critical points are summarized in the following result.

Proposition 3 For the family of order seven (3), the free critical points are:

$$
\begin{aligned}
\operatorname{cr}_{1}\left(\beta_{1}\right) & =-i, \\
\operatorname{cr}_{2}\left(\beta_{1}\right) & =i, \\
\operatorname{cr}_{3}\left(\beta_{1}\right) & =-\frac{1}{4} \sqrt{\frac{1}{-6+4 \beta_{1}}\left(57-\gamma+3 \sqrt{6} \varepsilon-2 \beta_{1}(9+\sqrt{6} \varepsilon)\right)} \\
& =-c r_{4}\left(\beta_{1}\right)=-\frac{1}{c r_{5}\left(\beta_{1}\right)}=\frac{1}{c r_{6}\left(\beta_{1}\right)}, \\
\operatorname{cr}_{9}\left(\beta_{1}\right) & =-\frac{1}{4} \sqrt{\frac{1}{-6+4 \beta_{1}}\left(57+\gamma-3 \sqrt{6} \varepsilon+2 \beta_{1}(-9+\sqrt{6} \varepsilon)\right)}=\frac{1}{c r_{7}\left(\beta_{1}\right)} \\
& =-c r_{10}\left(\beta_{1}\right)=-\frac{1}{c r_{8}\left(\beta_{1}\right)},
\end{aligned}
$$

where $\varepsilon=\sqrt{\frac{\theta}{\left(3-2 \beta_{1}\right)^{2}}}, \gamma=\sqrt{4977-9348 \beta_{1}+4420 \beta_{1}^{2}}, \theta=-165+108 \beta_{1}^{2}-19 \gamma+$ $2 \beta_{1}(74+3 \gamma)$ and $\delta=-165+108 \beta_{1}^{2}-19 \gamma+\beta_{1}(148-6 \gamma)$. Moreover,

(a) If $\beta_{1}=1$, then $\operatorname{cr}_{1}\left(\beta_{1}\right)=\operatorname{cr}_{3}\left(\beta_{1}\right)=-i$ and $\operatorname{cr}_{2}\left(\beta_{1}\right)=\operatorname{cr}_{4}\left(\beta_{1}\right)=i$. So, there are only six free critical points.

(b) If $\beta_{1}=\frac{16}{3}, \operatorname{cr}_{5}\left(\beta_{1}\right)=\operatorname{cr}_{7}\left(\beta_{1}\right)=-1$ and $\operatorname{cr}_{6}\left(\beta_{1}\right)=\operatorname{cr}_{8}\left(\beta_{1}\right)=1$. Then, there are only six free critical points.

Let us also remark that $c r_{1}\left(\beta_{1}\right)$ and $c r_{2}\left(\beta_{1}\right)$ are pre-images of $z=1$ and the following pairs are conjugated: $\operatorname{cr}_{3}\left(\beta_{1}\right)$ and $\operatorname{cr}_{5}\left(\beta_{1}\right), \operatorname{cr}_{4}\left(\beta_{1}\right)$ and $\operatorname{cr}_{6}\left(\beta_{1}\right), \operatorname{cr}_{7}\left(\beta_{1}\right)$ and $\operatorname{cr}_{9}\left(\beta_{1}\right), c r_{8}\left(\beta_{1}\right)$ and $c r_{10}\left(\beta_{1}\right)$. Therefore, due to the fact that the operator of the family has only pair powers, there are only two independent free critical points.

\subsection{The parameter and dynamical planes}

When we consider the free independent critical points of the family, we obtain the parameter plane $P_{2}$ (for $\left.c r_{i}\left(\beta_{1}\right), i=3,4,5,6\right)$ in Fig. 8a, b and $P_{3}$ for $c r_{i}\left(\beta_{1}\right), i=$ $7,8,9,10$, in Fig. $8 \mathrm{c}$, d. As it has been stated in the previous section, a mesh of 


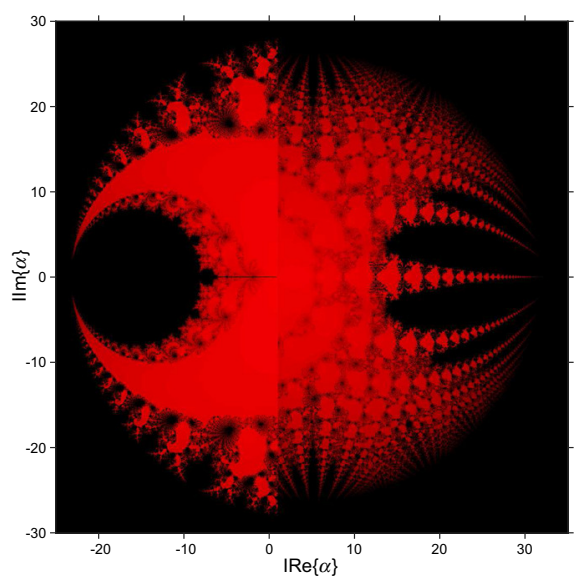

(a)

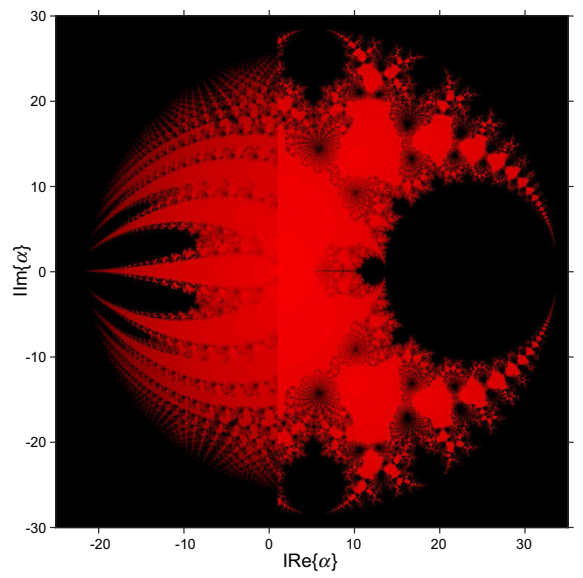

(c)

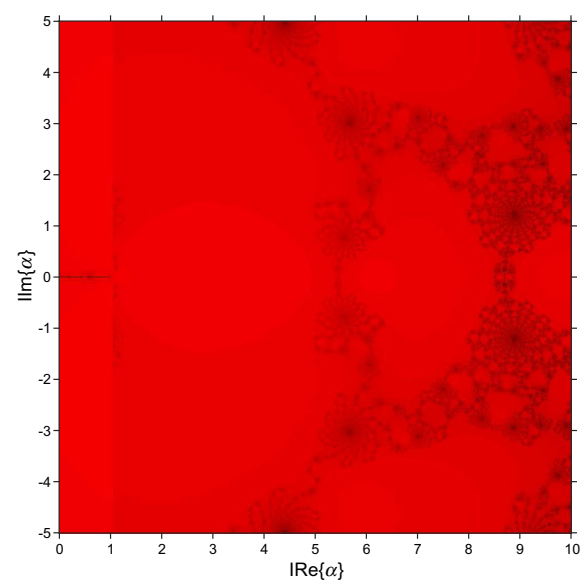

(b)

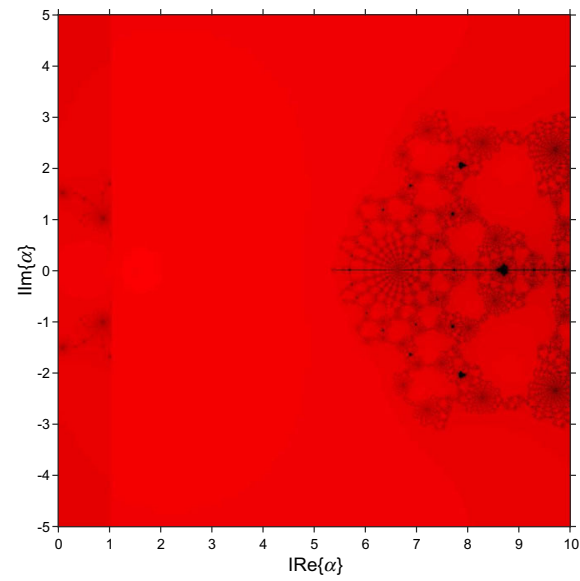

(d)

Fig. 8 Parameter planes of the family of order seven. a Parameter plane $P_{2}$, b parameter plane $P_{2}$ (a detail), c parameter plane $P_{3}$, d parameter plane $P_{3}$ (a detail)

$1000 \times 1000$ points has been used, the maximum number of iterations is 500 and the tolerance used has been $10^{-3}$ (in the software presented in [7]). Similarly as in the fourth-order family, the parameter space has reduced dimension because the operator of the family has only pair powers, that is its main advantage. Moreover, it can be observed in the detail of Fig. 8b that the region with stable behavior has increased its size with the increased order of convergence compared with Fig. 3. So, the best (clearest red areas) real values of the parameter $\beta_{1}$ are approximately located in $[1,6]$, with much wider areas of complex values with stable behavior. As a result, the number of best values of the parameter, in terms of the stability of the corresponding iterative methods, is bigger with order seven than order four.

Now, in Fig. 9, we show the dynamical planes with stable numerical behavior, corresponding to values of $\beta_{1}$ painted in red in the parameter planes. In this figure 


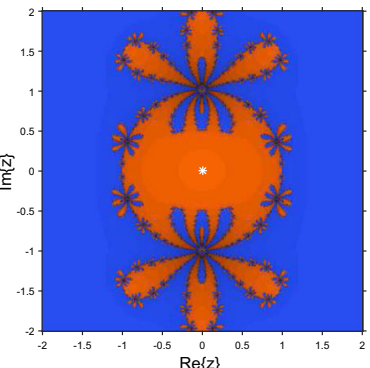

(a)

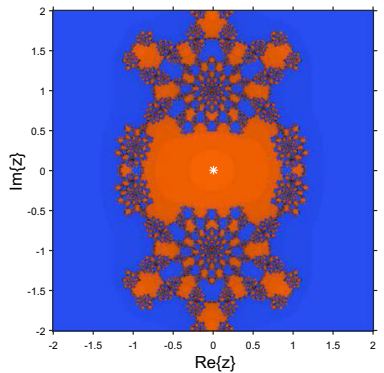

(d)

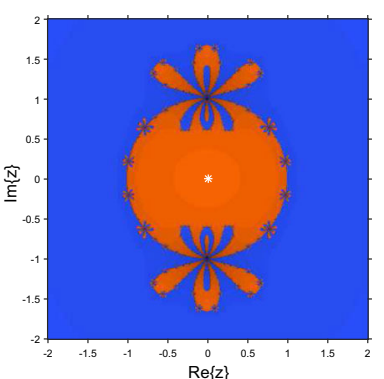

(b)

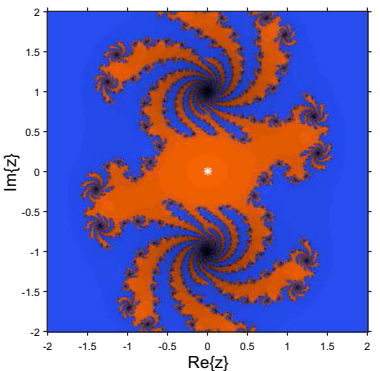

(e)

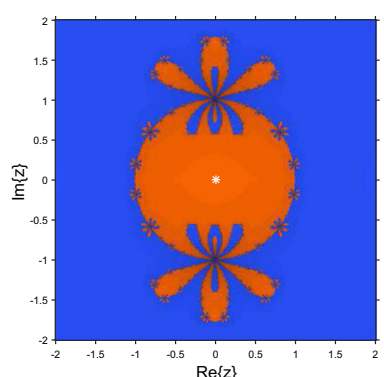

(c)

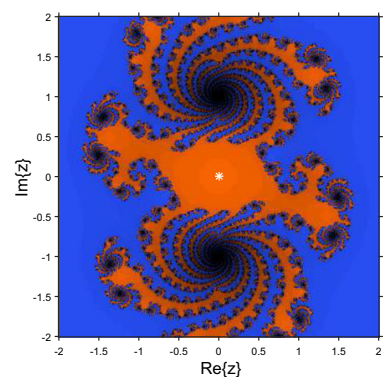

(f)

Fig. 9 Some dynamical planes with stable behavior. a $\beta_{1}=\frac{16}{3}, \mathbf{b} \beta_{1}=\frac{3}{2}$, c $\beta_{1}=2$, d $\beta_{1}=6$, e $\beta_{1}=2+10 i, \mathbf{f} \beta_{1}=-15 i$

different dynamical planes with only two basins of attraction appear: those of $z=0$ and $z=\infty$, that is, only convergence to the roots happens.

On the other hand, unstable behavior is found when we choose values of $\beta_{1}$ in some of the black regions of parameter planes.

Different kinds of unstable behavior can be found in Fig. 10: in Fig. 10a, two strange fixed points (whose basins of attraction appear in red and green, respectively) are attracting, meanwhile in Fig. 10e the parameter is inside the area of the complex plane where $e x_{1}\left(\beta_{1}\right)=1$ is slightly attracting and its basin is shown in green. The black color around the green one means that the initial estimations in this area need more that 40 iterations to reach the attracting strange fixed point. The rest of figures correspond to different periodic orbits painted in yellow color: in Fig. 10b the black region corresponds to the basin of attraction of a 2-periodic orbit; in Fig. 10c, d two orbits of period 5 and 6 , respectively, are shown. In the dynamical plane appearing in Fig. 10f a periodic orbit of period 3 appears; by applying Sharkovskii's Theorem, it is proved that there exist orbits of any period.

\section{Numerical performance}

Now, we are going to apply different elements of families (1) and (3) for solving an academic nonlinear system of arbitrary size. The values of parameters $\alpha_{1}$ and $\beta_{1}$ are 


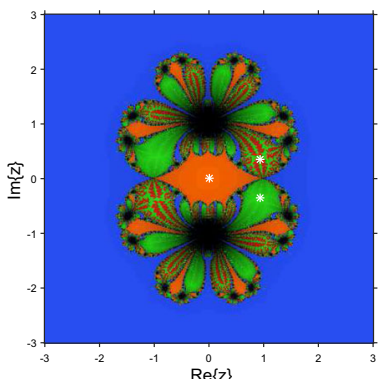

(a)

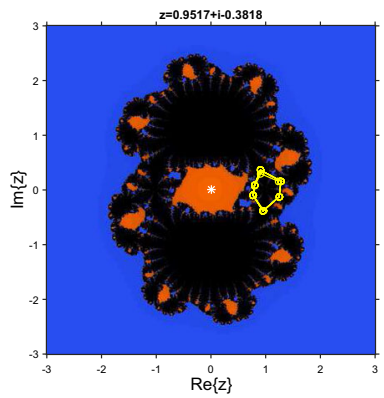

(d)

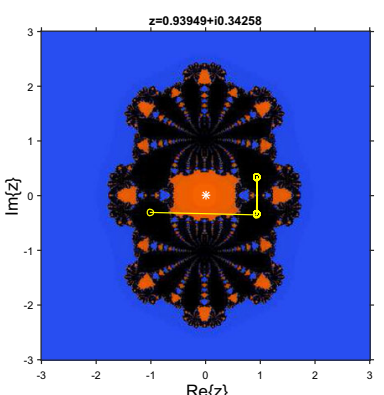

(b)

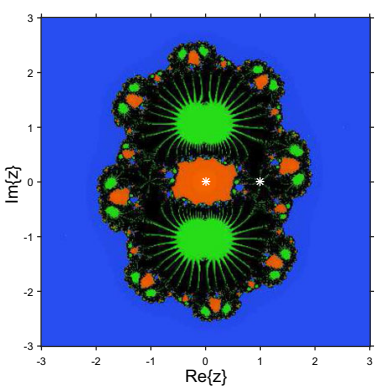

(e)

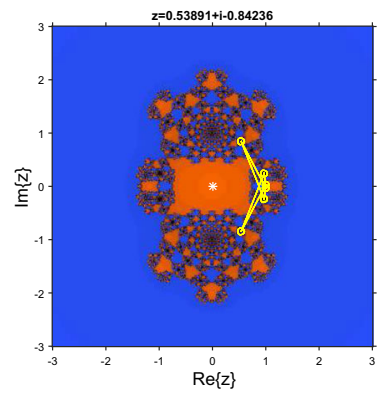

(c)

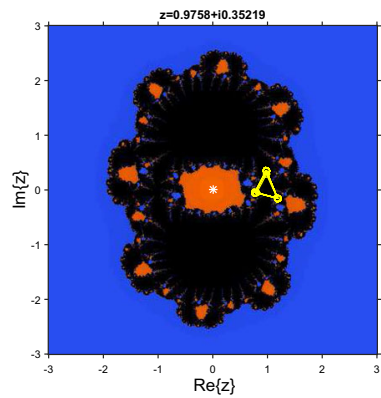

(f)

Fig. 10 Dynamical planes with unstable behavior. a $\beta_{1}=-15, \mathbf{b} \beta_{1}=20, \mathbf{c} \beta_{1}=8.75, \mathbf{d} \beta_{1}=-8+23 i$, e $\beta_{1}=20+25 i, \mathbf{f} \beta_{1}=19-23 i$

chosen from the dynamical results obtained in the previous sections. The numerical results have been obtained by using software Matlab 2015a, with variable precision arithmetics of 100 digits of mantissa and stoping criterium $\left\|F\left(x^{(k+1)}\right)\right\|<10^{-50}$ or $\left\|x^{(k+1)}-x^{(k)}\right\|<10^{-50}$.

We show, for each method, the number of iterations, the residual of the function at the last iteration, $\left\|F\left(x^{(k+1)}\right)\right\|$, the difference in norm between the two last iterations $\left\|x^{(k+1)}-x^{(k)}\right\|$ and the approximated computational order of convergence ACOC defined in [10] by

$$
p \approx A C O C=\frac{\ln \left(\left\|x^{(k+1)}-x^{(k)}\right\| /\left\|x^{(k)}-x^{(k-1)}\right\|\right)}{\ln \left(\left\|x^{(k)}-x^{(k-1)}\right\| /\left\|x^{(k-1)}-x^{(k-2)}\right\|\right)} .
$$

The value of $A C O C$ that appears in Tables 1 and 2 is the last coordinate of vector $A C O C$ when the variation between its values is small. Otherwise, it is marked with Example 1 Let us consider the nonlinear system of size $n \times n, n=20$,

$$
\left\{\begin{array}{l}
\left(x_{i} x_{i+1}\right)^{2}-3=0, \quad i=1,2, \ldots, n-1 \\
x_{n} x_{1}^{2}-1=0
\end{array}\right.
$$


Table 1 Numerical tests for Example 1 and family (1)

\begin{tabular}{lllll}
\hline Parameter & Iter & ACOC & $\left\|F\left(x^{(k+1)}\right)\right\|$ & $\left\|x^{(k+1)}-x^{(k)}\right\|$ \\
\hline$x^{(0)}=(3, \ldots, 3)^{T}$ & & & & \\
$\alpha_{1}=5 / 4$ & 4 & 4.762 & $5.55 \mathrm{e}-104$ & $8.16 \mathrm{e}-26$ \\
$\alpha_{1}=1$ & 5 & 3.9996 & $2.97 \mathrm{e}-107$ & $1.70 \mathrm{e}-43$ \\
$\alpha_{1}=2$ & 5 & 4.0079 & $1.48 \mathrm{e}-96$ & $1.50 \mathrm{e}-24$ \\
$\alpha_{1}=-3 / 2$ & 8 & 4.0101 & $5.11 \mathrm{e}-108$ & $4.79 \mathrm{e}-46$ \\
$\alpha_{1}=10$ & - & - & $>10^{6}$ & - \\
$\alpha_{1}=-20$ & - & - & $>10^{6}$ & - \\
$x^{(0)}=(0.8, \ldots, 0.8)^{T}$ & & & & \\
$\alpha_{1}=5 / 4$ & 5 & - & $1.70 \mathrm{e}-108$ & $1.36 \mathrm{e}-39$ \\
$\alpha_{1}=1$ & 5 & 4.0526 & $3.41 \mathrm{e}-108$ & $6.93 \mathrm{e}-27$ \\
$\alpha_{1}=2$ & - & - & $>10^{6}$ & - \\
$\alpha_{1}=-3 / 2$ & - & - & $>10^{6}$ & - \\
$\alpha_{1}=10$ & - & - & $>10^{6}$ & - \\
$\alpha_{1}=-20$ & - & - & $>10^{6}$ & - \\
\hline
\end{tabular}

The solution of this system obtained in any convergent case is $x^{*} \approx(0.575,0.575, \ldots$, $0.575)^{T}$.

In Table 1 we show the numerical results obtained for Example 1 by using some members of the fourth-order family (1) that have been presented in Sect. 2 as stable and unstable elements. Let us observe the bad numerical behavior for $\alpha_{1}=-3 / 2,10,-20$.

Similar results have been obtained for some members of seventh-order family (3) that correspond to stable $\left(\beta_{1}=3 / 2,2,6\right)$ and unstable $\left(\beta_{1}=-15,40,-20\right)$ cases, as can be seen in Table 2 .

In the following, we will show the performance of the best element of the fourthorder family (1) on a relevant chemical problem.

Example 2 An important problem in chemical engineering is to predict the diffusion and reaction in a porous catalyst pellet. The goal is to predict the overall reaction rate of the catalyst pellet. The conservation of mass in a spherical domain gives

$$
D\left[\frac{1}{r^{2}} \frac{d}{d r}\left(r^{2} \frac{d c}{d r}\right)\right]=k f(c), \quad 0<r<r_{p}
$$

where $r$ is the radial coordinate, $D$ the diffusivity, $c$ is the concentration of a given chemical, $k$ the rate constant and $f(c)$ the reaction rate function, and the conditions

$$
\frac{d c}{d r}(0)=0 \text { and } c\left(r_{p}\right)=c_{0}
$$

Now consider a sphere ( $5 \mathrm{~mm}$ in diameter) of $\gamma$-alumina upon which $P t$ is dispersed in order to catalyze the dehydrogenation of cyclohexane. At $700 \mathrm{~K}$, the rate constant 
Table 2 Numerical tests for Example 1 and family (3)

\begin{tabular}{lllll}
\hline Parameter & Iter & ACOC & $\left\|F\left(x^{(k+1)}\right)\right\|$ & $\left\|x^{(k+1)}-x^{(k)}\right\|$ \\
\hline$x^{(0)}=(3, \ldots, 3)^{T}$ & & & & \\
$\beta_{1}=3 / 2$ & 3 & 6.6805 & $1.15 \mathrm{e}-68$ & $7.11 \mathrm{e}-12$ \\
$\beta_{1}=2$ & 3 & 5.5302 & $2.41 \mathrm{e}-68$ & $7.17 \mathrm{e}-12$ \\
$\beta_{1}=6$ & 4 & - & $3.41 \mathrm{e}-108$ & $8.37 \mathrm{e}-29$ \\
$\beta_{1}=-15$ & 5 & 6.2807 & $3.41 \mathrm{e}-108$ & $5.51 \mathrm{e}-36$ \\
$\beta_{1}=40$ & 6 & - & $3.41 \mathrm{e}-108$ & $1.74 \mathrm{e}-34$ \\
$\beta_{1}=-20$ & 5 & - & $1.70 \mathrm{e}-108$ & $1.57 \mathrm{e}-20$ \\
$x^{(0)}=(0.8, \ldots, 0.8)^{T}$ & & & & \\
$\beta_{1}=3 / 2$ & 4 & - & $2.97 \mathrm{e}-107$ & $6.88 \mathrm{e}-27$ \\
$\beta_{1}=2$ & 4 & - & $2.97 \mathrm{e}-107$ & $2.95 \mathrm{e}-25$ \\
$\beta_{1}=6$ & - & - & $>10^{6}$ & - \\
$\beta_{1}=-15$ & - & - & $>10^{6}$ & - \\
$\beta_{1}=40$ & - & - & $>10^{6}$ & - \\
$\beta_{1}=-20$ & - & - & $>10^{6}$ & - \\
\hline
\end{tabular}

$k$ is $4 \mathrm{~s}^{-1}$, and the diffusivity D is $5 \times 10^{-2} \mathrm{~cm}^{2} / \mathrm{s}$. Set up the equations necessary to calculate the concentration profile of cyclohexane within the pellet and also the effectiveness factor for a general $f(c)$. Next, solve these equations for $f(c)=c^{2}$.

We define

$$
C=\frac{\text { concentration of cyclohexane }}{\text { concentration of cyclohexane at the surface of the sphere }}
$$

and $R=$ dimensionless radial coordinate based on the radius of the sphere $\left(r_{p}=2.5\right.$ $\mathrm{mm}$ ).

Let us assume that the spherical pellet is isothermal. The conservation of mass equation for cyclohexane is

$$
\frac{d^{2} C}{d R^{2}}+\frac{2}{R} \frac{d C}{d R}=\Phi^{2} C^{2}, \quad 0<R<1,
$$

with conditions

$$
\frac{d C}{d R}(0)=0, \quad C(1)=1,
$$

where

$$
\Phi=r_{p} \sqrt{\frac{k}{D}}, \quad \text { (Thiele modulus) }
$$

that, in this case is $\Phi=2.236$. 
By using central divided differences, we transform the boundary value problem (4) in a system of nonlinear equations, which will be solved by applying the methods object of this work. We use

$$
C^{\prime \prime}(R) \approx \frac{C(R+h)-2 C(R)+C(R-h)}{h^{2}}, \quad C^{\prime}(R) \approx \frac{C(R+h)-C(R-h)}{2 h},
$$

where $h=\frac{1}{n+1}$ is the mesh spacing. If we denote by $C_{i}=C\left(R_{i}\right)$, with $R_{i}=0+i h$, $i=0,1, \ldots, n+1$, the mesh points, the boundary value problem can be approximated by the nonlinear system

$$
\left(1+\frac{1}{R_{i}}\right) C_{i+1}-2 C_{i}+\left(1-\frac{1}{R_{i}}\right) C_{i-1}=h^{2} \Phi^{2} C_{i}^{2}, \quad i=1,2, \ldots, n
$$

with $C_{n+1}=1$. For $R=0$, the second term in the differential equation is evaluating taking into account that

$$
\lim _{R \rightarrow 0} \frac{C^{\prime}}{R}=C^{\prime \prime}
$$

so, the differential equation becomes $3 C^{\prime \prime}-\Phi^{2} C^{2}=0$. Therefore, the corresponding difference replacement is

$$
C_{1}-2 C_{0}+C_{-1}-\frac{1}{3} h^{2} \Phi^{2} C_{0}^{2}=0 .
$$

Using central divided differences in the boundary condition $C^{\prime}(0)=0$ we obtain that $C_{1}=C_{-1}$, so the first equation of our system is $C_{1}-C_{0}-\frac{1}{6} h^{2} \Phi^{2} C_{0}^{2}=0$.

Problem (4) has been approximated by the nonlinear system $F(C)=0$, where $F: \mathbb{R}^{n+1} \rightarrow \mathbb{R}^{n+1}$ is defined by

$$
F(C)=\left\{\begin{array}{l}
C_{1}-C_{0}-\frac{1}{6} h^{2} \Phi^{2} C_{0}^{2}, \\
\vdots \\
(1+1 / i) C_{i+1}-2 C_{i}+(1-1 / i) C_{i-1}-h^{2} \Phi^{2} C_{i}^{2}, \quad i=1,2, \ldots, n-1 \\
\vdots \\
1+1 / n-2 C_{n}+(1-1 / n) C_{n-1}-h^{2} \Phi^{2} C_{n}^{2},
\end{array}\right.
$$

whose Jacobian matrix is

$$
F^{\prime}(C)=\left(\begin{array}{cccccc}
a_{0} & d_{0} & 0 & \ldots & 0 & 0 \\
b_{1} & a_{1} & d_{1} & \ldots & 0 & 0 \\
0 & b_{2} & a_{2} & \ldots & 0 & 0 \\
\vdots & \vdots & \vdots & & \vdots & \vdots \\
0 & 0 & 0 & \ldots & a_{n-1} & d_{n-1} \\
0 & 0 & 0 & \ldots & b_{n} & a_{n}
\end{array}\right)
$$


Table 3 Solution for different size of the system

\begin{tabular}{llll}
\hline $\mathrm{R}$ & $n=10$ & $n=20$ & $n=100$ \\
\hline 0.0 & 0.5934 & 0.5924 & 0.5921 \\
0.2 & 0.6053 & 0.6043 & 0.6039 \\
0.4 & 0.6425 & 0.6415 & 0.6412 \\
0.6 & 0.7108 & 0.7099 & 0.7096 \\
0.8 & 0.8223 & 0.8216 & 0.8214 \\
1.0 & 1.0 & 1.0 & 1.0 \\
\hline
\end{tabular}

where

$$
\begin{aligned}
a_{0} & =-1-(1 / 3) h^{2} \Phi^{2} C_{0}, \quad d_{0}=1 \\
b_{i} & =1-1 / i, i=1,2, \ldots, n \\
a_{i} & =-2-2 h^{2} \Phi^{2} C_{i}, i=1,2, \ldots, n \\
d_{i} & =1+1 / i, i=1,2, \ldots, n-1
\end{aligned}
$$

In Table 3, we show the approximated result for some values of $R$, using the element of family (1) corresponding to $\alpha_{1}=5 / 4$ and different sizes of the system. We use the initial estimation $x^{(0)}=(0.5,0.5, \ldots, 0.5)^{T}$ and in any case the method has converged to the presented solution in three iterations.

\section{Conclusions}

A dynamical study on quadratic polynomials of two parametric families of iterative methods for solving nonlinear problems has been presented, in order to detect their most stable elements or those with bad stability properties. From the parameter planes associated to both classes, it has been proved that there are more values of the parameter, that is, elements of the family, with good stability properties when we increase the order of this family. About the family of order seven, we have observed in the parameter plane that unstable values of the parameter are located in small and sparse regions of the complex plane. Except in these small regions of the parameter planes, the behavior of schemes in the class is very stable. These results have been numerically checked on an academic example and on the chemical problem of predicting the diffusion and reaction in a porous catalyst pellet.

\section{References}

1. S. Amat, S. Busquier, Advances in Iterative Methods for Nonlinear Equations (Springer, Berlin, 2016)

2. S. Amat, S. Busquier, S. Plaza, Review of some iterative root-finding methods from a dynamical point of view. Sci. Ser. A Math. Sci. 10, 3-35 (2004)

3. S. Amat, S. Busquier, S. Plaza, A construction of attracting periodic orbits for some classical third-order iterative methods. Comput. Appl. Math. 189, 22-33 (2006)

4. I.K. Argyros, Á.A. Magreñn, On the convergence of an optimal fourth-order family of methods and its dynamics. Appl. Math. Comput. 252, 336-346 (2015) 
5. D.K.R. Babajee, A. Cordero, J.R. Torregrosa, Study of multipoint iterative methods through the Cayley quadratic test. Comput. Appl. Math. 291, 358-369 (2016). doi:10.1016/J.CAM.2014.09.020

6. P. Blanchard, The dynamics of Newton's method. Proc. Symp. Appl. Math. 49, 139-154 (1994)

7. F.I. Chicharro, A. Cordero, J.R. Torregrosa, Drawing dynamical and parameters planes of iterative families and methods. Sci. World J. 2013, Article ID 780153 (2013)

8. C. Chun, M.Y. Lee, B. Neta, J. Džunić, On optimal fourth-order iterative methods free from second derivative and their dynamics. Appl. Math. Comput. 218, 6427-6438 (2012)

9. A. Cordero, E. Gómez, J.R. Torregrosa, Efficient high-order iterative methods for solving nonlinear systems and their application on heat conduction problems. Complexity 2017, Article ID 6457532 (2017)

10. A. Cordero, J.R. Torregrosa, Variants of Newton's method using fifth-order quadrature formulas. Appl. Math. Comput. 190, 686-698 (2007)

11. R.L. Devaney, An Introduction to Chaotic Dynamical Systems (Addison-Wesley Publishing Company, Reading, 1989)

12. P.G. Logrado, J.D.M. Vianna, Partitioning technique procedure revisited: formalism and first application to atomic problems. Math. Chem. 22, 107-116 (1997)

13. C.G. Jesudason, I. Numerical nonlinear analysis: differential methods and optimization applied to chemical reaction rate determination. Math. Chem. 49, 1384-1415 (2011)

14. Á.A. Magreñán, Different anomalies in a Jarratt family of iterative root-finding methods. Appl. Math. Comput. 233, 29-38 (2014)

15. M. Mahalakshmi, G. Hariharan, K. Kannan, The wavelet methods to linear and nonlinear reactiondiffusion model arising in mathematical chemistry. Math. Chem. 51(9), 2361-2385 (2013)

16. K. Maleknejad, M. Alizadeh, An efficient numerical scheme for solving Hammerstein integral equation arisen in chemical phenomenon. Proc. Comput. Sci. 3, 361-364 (2011)

17. B. Neta, C. Chun, M. Scott, Basins of attraction for optimal eighth-order methods to find simple roots of nonlinear equations. Appl. Math. Comput. 227, 567-592 (2014)

18. M.S. Petković, B. Neta, L.D. Petković, J. Džunić, Multipoint Methods for Solving Nonlinear Equations (Elsevier, Amsterdam, 2013)

19. R.C. Rach, J.S. Duan, A.M. Wazwaz, Solving coupled Lane-Emden boundary value problems in catalytic diffusion reactions by the Adomian decomposition method. Math. Chem. 52(1), 255-267 (2014)

20. R. Singh, G. Nelakanti, J. Kumar, A new effcient technique for solving two-point boundary value problems for integro-differential equations. Math. Chem. 52, 2030-2051 (2014) 


\section{Author Query Form}

\section{Please ensure you fill out your response to the queries raised below and return this form along with your corrections}

\section{Dear Author}

During the process of typesetting your article, the following queries have arisen. Please check your typeset proof carefully against the queries listed below and mark the necessary changes either directly on the proof/online grid or in the 'Author's response' area provided below

\begin{tabular}{|c|l|l|}
\hline Query & \multicolumn{1}{|c|}{ Details required } & Author's response \\
\hline 1. & $\begin{array}{l}\text { Please check and confirm the edit } \\
\text { made in article title. }\end{array}$ & \\
\hline 2. & $\begin{array}{l}\text { Please confirm the corresponding } \\
\text { author is correctly identified and } \\
\text { amend if necessary. }\end{array}$ & \\
\hline 3. & $\begin{array}{l}\text { Please check and confirm the inserted } \\
\text { citation of Fig. } 3 \text { is correct. If not, } \\
\text { please suggest an alternative citation. } \\
\text { Please note that figures should be cited } \\
\text { in sequential order in the text. }\end{array}$ & \\
\hline 4. & $\begin{array}{l}\text { As per the information provided by } \\
\text { the publisher, Figs. } 4,3,7,9,10 \text { would } \\
\text { be black and white in print; hence, } \\
\text { please confirm whether we can add } \\
\text { "colour figure online" as mentioned } \\
\text { in the text. }\end{array}$ & \\
\hline 5. & $\begin{array}{l}\text { Kindly check and confirm the inserted } \\
\text { publication year are correct for the ref- } \\
\text { erence [7]. }\end{array}$ & \\
\hline
\end{tabular}

